\title{
INTELIGENCIA EMOCIONAL Y ESTRÉS ACADÉMICO EN ESTUDIANTES DE ENFERMERÍA
}

\author{
EMOTIONAL INTELLIGENCE AND ACADEMIC STRESS IN NURSING \\ STUDENTS
}

\author{
INTELIGÊNCIA EMOCIONAL E ESTRESSE ACADÊMICO EM \\ ESTUDANTES DE ENFERMAGEM
}

\author{
Mónica Quiliano Navarro* \\ Miryam Quiliano Navarro**
}

\begin{abstract}
RESUMEN
Objetivo: Determinar la relación que existe entre inteligencia emocional y estrés académico de los estudiantes de enfermería. Material y Método: Estudio ex post facto, descriptivo, transversal en una Facultad de Enfermería de una universidad peruana. De una población de 320 estudiantes de Enfermería, matriculados en VII semestre, se obtuvo una muestra no probabilística de 30 discentes que consintieron informadamente a participar. Se emplearon 2 instrumentos: Uno elaborado por Bar-On, validado en Perú para medir Inteligencia Emocional y el Inventario SISCO para Estrés académico. Para medidas psicométricas se empleó el alfa de Cronbach y análisis descriptivo en tablas de distribución de frecuencias, medidas de tendencia central y dispersión. Para buscar asociación entre Inteligencia emocional y Estrés académico se utilizó Chi-cuadrado $\left(\mathrm{X}^{2}\right)$. El estudio fue aprobado por el Comité de Ética del Instituto de Investigación de la Facultad de Enfermería. Resultados: Los estudiantes poseen escaza inteligencia emocional en relación con las dimensiones Intrapersonal y Adaptabilidad; las dimensiones Inteligencia interpersonal y Estado de ánimo general son las más desarrolladas en ellos. Las cinco dimensiones de la Inteligencia emocional están relacionadas con episodios de estrés en estudiantes que alguna vez presentaron esta situación. Conclusiones: No se encontró asociación entre las dimensiones de la Inteligencia emocional y la frecuencia del Estrés académico en estudiantes de Enfermería del VII semestre. Es necesario trabajar por mejorar la calidad educativa durante el proceso de formación profesional y considerar el aspecto emocional para desarrollar un proceso enseńanza-aprendizaje integral.
\end{abstract}

Palabras clave: Inteligencia emocional; Estrés académico; Estudiante de enfermería; Habilidades sociales.

\section{ABSTRACT}

Objective: To determine the relationship between emotional intelligence and academic stress among nursing students. Material and Method: Ex post facto, descriptive, cross-sectional study carried out in the Faculty of Nursing of a Peruvian University. A non-probabilistic sample of 30 students, who informedly consented

*Enfermera, Doctora en Ciencias de la Salud y Salud Pública, Docente de la Facultad de Enfermería, Universidad Nacional del Centro del Perú, Huancayo, Perú. Email: mquiliano@uncp.edu.pe. Autor de correspondencia.

**Enfermera, Especialista en Salud Pública, Docente de la Facultad de Enfermería, Universidad Norbert Wiener, Lima, Perú. Email: mquiliano@hotmail.es 
to participate, was obtained from a population of 320 nursing students enrolled in the $7^{\text {th }}$ semester. Two instruments were used: one prepared by Bar-On, validated for Peru to measure emotional intelligence and the SISCO Inventory for Academic Stress. For psychometric measurements, Cronbach's alpha, descriptive analysis in frequency distribution tables, central tendencies and dispersion measurements were used. To establish a relationship between emotional intelligence and academic stress, Chi-squared $\left(\mathrm{X}^{2}\right)$ test was used. The study was approved by the Ethics Committee of the Research Institute of the Faculty of Nursing. Results: The students showed a lack of Emotional intelligence in relation to the Intrapersonal and Adaptability dimensions. The Interpersonal intelligence and general mood are the most developed. The five dimensions of Emotional intelligence are related to stress episodes of students who have been affected by this situation. Conclusions: There is no significant relationship between emotional intelligence and frequency of academic stress among nursing students in the $7^{\text {th }}$ semester. Nevertheless, it is necessary to work in order to improve the educational quality during the process of professional training and to consider the emotional component to develop a comprehensive teaching and learning process.

Keywords: Emotional Intelligence; Academic Stress; Nursing Students; Social Skills.

\section{RESUMO}

Objetivo: Determinar a relação existente entre inteligência emocional e estresse acadêmico dos estudantes de enfermagem. Material e Método: Estudo ex post facto, descritivo, transversal na Faculdade de Enfermagem de uma universidade peruana. De uma população de 320 estudantes de Enfermagem matriculados no semestre VII, foi obtida uma amostra não probabilística de 30 estudantes que foram informados e consentiram sua participação. Foram utilizados 2 instrumentos: Um preparado pelo Bar-On, validado para o Peru para medir Inteligência Emocional e o inventário SISCO para Estresse acadêmico. Para medidas psicométricas foi usado o alfa de Cronbach, análise descritiva em tabelas de distribuição de frequências, medidas de tendência central e dispersão. Para encontrar uma associação entre Inteligência Emocional e Estresse acadêmico utilizou-se o Chi ${ }^{2}$. O estudo foi aprovado pelo Comitê de Ética do Instituto de Pesquisa da Faculdade de Enfermagem. Resultados: Os estudantes têm pouca Inteligência emocional em relação às dimensôes Intrapessoais e Adaptabilidade; a Inteligência interpessoal e as dimensóes gerais do humor são as mais desenvolvidas. As cinco dimensôes da Inteligência emocional estão relacionadas com episódios de estresse em estudantes que já apresentaram essa situação. Conclusóes: Não foi encontrada associação entre as dimensôes da Inteligência Emocional e o Estresse acadêmico em Estudantes de Enfermagem do semestre VII. É preciso trabalhar para melhorar a qualidade da educação durante o processo de formação profissional e considerar o aspecto emocional para desenvolver um processo abrangente de ensino-aprendizagem.

Palavras-chave: Inteligência emocional; Estresse acadêmico; Estudante de enfermagem; Habilidades sociais.

Fecha de recepción: 20/05/2019

Fecha de aceptación: 04/10/2019

\section{INTRODUCCIÓN}

Vivimos en un mundo globalizado, con diversos enfoques en todos los ámbitos del quehacer del ser humano. El proceso enseñanza-aprendizaje no es ajeno a esto, está sujeto a múltiples reformas, generando impactos inequívocos y rápidos, con consecuencias de largo recorrido que son difíciles de prever. En este contexto, el proceso de formación profesional del discente universitario es una experiencia de aprendizaje, que por lo general está acompañado de Estrés académico (EA) a causa de un inadecuado desarrollo de habilidades que estimulan su Inteligencia Emocional (IE).

La IE se define como la capacidad para razonar sobre las emociones, refleja la capacidad del individuo para hacer mejor uso de sus propias cualidades y competencias sociales para el desarrollo individual, emocional, intelectual y profesional ${ }^{(1)}$. Goleman $^{(2)}$ refiere que la IE proporciona al 
individuo "una ventaja en cualquier ámbito de la vida, sea en las relaciones románticas e íntimas o captando las reglas tácitas que gobiernan el éxito en la política organizacional". La IE está relacionada con el éxito académico ${ }^{(3)}$, y también con el ajuste psicológico, social, o la satisfacción personal y el bienestar psicológico ${ }^{(4)}$.

Las destrezas emocionales deben ser aplicadas en diversos contextos y en cualquier circunstancia, con la finalidad de formar personas emocionalmente inteligentes. Márquez y Gaeta ${ }^{(5)}$ afirman que la familia participa en el desarrollo humano y personal de todos sus integrantes, contribuyendo también con el desarrollo de distintas competencias de la IE. Las competencias emocionales adquiridas en el contexto familiar son primordiales y trascendentes para ser usadas en cualquier tipo de contexto. Además, la IE juega un papel muy importante en el proceso enseñanza-aprendizaje y un desarrollo de emociones negativas limita dicho proceso ${ }^{(6)}$. En consecuencia, el desarrollo de la IE en estudiantes universitarios es fundamental para el progreso académico y personal.

Coincidiendo con Farquhar, Kamei y Vidyarthi ${ }^{(7)}$, se puede aseverar que el estudio de las emociones puede contribuir con datos importantes para el discente y profesorado. En últimas investigaciones se considera que la IE juega un rol importante en el proceso enseńanza-aprendizaje de los estudiantes de ciencias de la salud, tanto para la salud mental como para la práctica profesional, ya que el EA, en los estudiantes de estas áreas, se presenta en altos niveles, y la IE, así como la resiliencia, pueden ser consideradas como amortiguadoras de este estrés y del agotamiento de los estudiantes.

Algunos autores manifiestan que las personas con alto grado de IE tienen menos reacciones psicofisiológicas frente al estrés, conocen bien sus sentimientos y emociones, y son conscientes de cómo sus reacciones pueden afectar a otras personas ${ }^{(8)}$. Así mismo, se ha propuesto que una elevada IE suministra más competencias para afrontar el estrés, puesto que ésta regula todo el material potencialmente nocivo para el cuerpo, facilitando el reconocimiento de emociones y sentimientos que permiten tomar decisiones adecuadas frente a situaciones difíciles ${ }^{(9)}$. De acuerdo con los razonamientos anteriores, las personas con IE alta son más eficientes en sortear circunstancias que pueden producir situaciones estresantes.

Existe evidencia en la literatura que la IE es un fenómeno factible de desarrollar en el ser humano, tiende a aumentar con la edad y con programas de capacitación sistemáticos. Así mismo, existen investigaciones que muestran una correlación entre IE y logro académico ${ }^{(10)}$. Lo mencionado sugiere la necesidad de realizar investigaciones dirigidas a solucionar brechas evidenciadas durante el proceso de enseñanza-aprendizaje del estudiante universitario. Otro estudio demostró la correlación entre IE y satisfacción personal, bienestar psicológico, ajuste social, y satisfacción con la vida, declarando que el bienestar de los estudiantes es necesario para que puedan desempeñarse de manera adecuada en su formación ${ }^{(11)}$. Aun así, existen escasos estudios que aborden la relación entre IE y EA. Algunas investigaciones realizadas en discentes universitarios de primeros semestres ${ }^{(12)}$ sugieren realizar estudios en niveles superiores, pues éstos enfrentan situaciones de mayor responsabilidad y experimentan estrés con un desarrollo emocional inadecuado.

En este contexto, los estudiantes de ciencias de la salud tienen niveles de EA significativamente mayores que los estudiantes que cursan otras disciplinas $^{(13)}$, con un incremento significativo durante la fase de formación teórica y de experiencia práctica que se realiza en el ambiente clínicoasistencial.

Según lo anterior, el objetivo de esta investigación fue determinar la relación que existe entre IE y EA en estudiantes universitarios de una Facultad de Enfermería, puesto que el estrés experimentado por ellos, en el contexto académico, constituye un tópico de gran interés, debido a sus implicaciones en el rendimiento, aprendizaje, salud y bienestar. Debido a la naturaleza transversal de este estudio, esta conjetura solo puede interpretarse con ayuda de un referente teórico, por tanto, no es posible establecer relación causal entre las variables de estudio. Esta investigación se ajusta al modelo cognitivo transaccional del estrés, donde los estímulos externos, los recursos de afrontamiento con los que cuenta el individuo y el manejo adecuado de la IE determinan la valoración estresante de un evento, las estrategias para su manejo y las repercusiones sobre su estado físico, psicológico y social. 


\section{MATERIAL Y MÉTODO}

Tipo de estudio y unidad de análisis: El estudio fue ex post facto, de tipo descriptivo y transversal realizado con estudiantes de Enfermería de la Facultad de Enfermería de una universidad peruana.

Plan de muestreo: La población estuvo conformada por 320 estudiantes y la muestra, por conveniencia, fue de 30 discentes que firmaron el consentimiento informado y llenaron adecuadamente la encuesta. Como criterios de inclusión se consideró a estudiantes matriculados en el VII semestre y en condición de estudiantes regulares. El criterio de exclusión fue la no asistencia a clases el día que se aplicó la encuesta.

Recolección de datos: Fue mediante encuesta, a través de cuestionarios estructurados que fueron aplicados en una sola sesión bajo la supervisión directa de las investigadoras. Se explicó a los estudiantes el objetivo del estudio, la forma de responder los cuestionarios, y el carácter confidencial de la información obtenida. El tiempo destinado para el llenado de los cuestionarios osciló entre 15 y 20 min. Para medir Inteligencia Emocional, se empleó el instrumento elaborado por Bar-On, adecuado y validado por Chávez y Águila ${ }^{(14)}$ para la realidad peruana. Este instrumento consigna 15 subcomponentes que están distribuidos en cinco dimensiones: Inteligencia interpersonal, Inteligencia intrapersonal, Adaptabilidad, Manejo de estrés y Estado de ánimo en general.

También se utilizó el Inventario SISCO para medir Estrés Académico, creado por Barraza ${ }^{(15)}$ y conformado por 31 ítems que miden: Intensidad de estrés autopercibido, Entorno estresor, Reacciones y Estrategias de afrontamiento utilizadas por los estudiantes ante el estrés. Uno de los ítems es considerado como filtro y otro valora el nivel de intensidad de estrés académico. Del total, 29 ítems contemplan un escalamiento tipo Likert, 8 identifican la frecuencia en que las demandas del entorno son valoradas como estímulos estresores, 15 identifican la frecuencia de los síntomas o reacciones al estímulo estresor y 6 identifican la frecuencia de uso de estrategias de afrontamiento.

Confiabilidad de los instrumentos: Se obtuvo la propiedad psicométrica de fiabilidad de los cuestionarios por medio del coeficiente de alfa de Cronbach. La consistencia interna para el inventario de IE fue muy alta $(0,93)$ y lo mismo ocurrió con el Inventario SISCO $(0,90)$.

Análisis de datos: Se realizó el análisis descriptivo de cada variable mediante tablas de distribución de frecuencias, medidas de tendencia central y dispersión, utilizando SPSS versión 20. Para buscar asociación entre las dimensiones de la IE y la frecuencia del EA se realizó un análisis bivariado por medio de la prueba estadística Chi-cuadrado $\left(\mathrm{X}^{2}\right)$.

La investigación siguió las consideraciones éticas nacionales e internacionales, siendo aprobado por el Instituto de Investigación y el Comité de Ética de la Facultad de Enfermería.

\section{RESULTADOS}

Inteligencia emocional: En la Tabla 1, en correspondencia a la Inteligencia interpersonal, la mayor frecuencia la obtuvo la categoría Promedio (47\%); en la Inteligencia intrapersonal, la mayor frecuencia fue el nivel Muy bajo (43\%); en la Adaptabilidad el nivel con mayor incidencia fue Muy (37\%); en el Manejo de estrés fue más frecuente el nivel Bajo (47\%) y, por último, el Estado de ánimo general obtuvo mayor frecuencia en el nivel Promedio (37\%). Según lo anterior, los estudiantes del estudio, al parecer, no han desarrollado una adecuada IE en sus distintas dimensiones, lo cual limitaría la cantidad y calidad de redes interpersonales y de apoyo social, además de las dificultades para enfrentar situaciones de EA.

Estrés académico: La Tabla 2 señala que el 63\% de los estudiantes presentó alguna vez este tipo de estrés, con un promedio de intensidad alta $(81,3)$, lo cual puede influir de manera negativa en su estado de salud, su aprendizaje y desempeño académico. En la Tabla 3 se observa la evaluación de las características del EA.

Inteligencia emocional y Estrés académico: La Tabla 4 evidencia que las cinco dimensiones de la IE están más representadas en 19 de los estudiantes que alguna vez presentaron un episodio de estrés, pero a pesar de ello, no se encontró asociación estadísticamente significativa entre ninguna de estas dimensiones y la frecuencia de EA. 


\section{DISCUSIÓN}

La no asociación entre los niveles de IE y la frecuencia de EA discrepan con los resultados de Bryant y Malone ${ }^{(16)}$, quienes afirman que los estudiantes con incremento de los niveles de IE experimentan niveles más bajos de estrés. Así mismo, los resultados del estudio no coinciden con lo descrito por Cabanach, Souto y Fernández ${ }^{(13)}$, quienes encontraron que los estudiantes con elevadas puntuaciones en control y aceptación de sus estados emocionales perciben las circunstancias académicas de forma más adaptativa y experimentan menores respuestas de estrés. De igual forma Barraza, Muñoz y Behrens ${ }^{(9)}$ encontraron que la IE percibida por estudiantes de medicina reportaba, en promedio, niveles adecuados para la percepción, comprensión y manejo de las emociones. Por otro lado, los resultados de esta investigación sí coinciden con Picasso, Lizano y Anduaga ${ }^{(17)}$, quienes concluyeron que la mayoría de los estudiantes presentan momentos de estrés durante el transcurso del semestre y la mayoría presenta una percepción deficiente de sus emociones por lo que deben mejorar respecto a la comprensión y la regulación de las mismas. Lo anterior confirma que el inadecuado desarrollo de la IE podría estar limitando la cantidad y calidad de redes de apoyo social de los estudiantes de Enfermería y, como consecuencia, puede ser más problemático para ellos enfrentar situaciones de estrés durante el proceso enseńanza-aprendizaje.

Tabla 1. Inteligencia Emocional de los estudiantes de Enfermería del VII semestre ( $\mathrm{n}=30)$.

\begin{tabular}{|c|c|c|c|c|c|c|c|c|c|c|c|c|c|c|c|c|}
\hline \multirow{2}{*}{$\begin{array}{l}\text { Dimensión Inteligencia } \\
\text { Emocional }\end{array}$} & \multicolumn{2}{|c|}{$\begin{array}{c}\text { Marcd } \\
\text { Bajo }\end{array}$} & \multicolumn{2}{|c|}{$\begin{array}{l}\text { Muy } \\
\text { bajo }\end{array}$} & \multicolumn{2}{|c|}{ Bajo } & \multicolumn{2}{|c|}{ Promedio } & \multicolumn{2}{|c|}{ Alto } & \multicolumn{2}{|c|}{$\begin{array}{l}\text { Muy } \\
\text { Alto }\end{array}$} & \multicolumn{2}{|c|}{$\begin{array}{c}\text { Marcd } \\
\text { Alto }\end{array}$} & \multicolumn{2}{|c|}{ Total } \\
\hline & $\mathbf{N}^{\circ}$ & $\%$ & $\mathbf{N}^{\circ}$ & $\%$ & $\mathbf{N}^{\circ}$ & $\%$ & $\mathbf{N}^{\circ}$ & $\%$ & $\mathbf{N}^{\circ}$ & $\%$ & $\mathbf{N}^{\circ}$ & $\%$ & $\mathbf{N}^{\circ}$ & $\%$ & $\mathbf{N}^{\circ}$ & $\%$ \\
\hline & 2 & 7 & 3 & 10 & 6 & 20 & 14 & & 4 & 13 & 1 & 3 & 0 & 0 & 30 & 100 \\
\hline Inteligencia Intrapersonal & 12 & 40 & 13 & 43 & 3 & 10 & 2 & 7 & 0 & 0 & 0 & 0 & 0 & 0 & 30 & 100 \\
\hline & 3 & 10 & 11 & 37 & 10 & 33 & 5 & 17 & 1 & 3 & 0 & 0 & 0 & 0 & 30 & 100 \\
\hline & 0 & 0 & 4 & 13 & 14 & 47 & 9 & 30 & 3 & 10 & 0 & 0 & 0 & 0 & 30 & 100 \\
\hline Estado de ánimo en general & 6 & 20 & 5 & 17 & 7 & 23 & 11 & 37 & 1 & 3 & 0 & 0 & 0 & 0 & 30 & 100 \\
\hline
\end{tabular}

Tabla 2. Frecuencia de Estrés Académico en estudiantes regulares de Enfermería del VII semestre (n=30).

\begin{tabular}{lrr}
\hline Frecuencia de estrés & Fr & (\%) \\
\hline Siempre & 2 & 7 \\
Casi siempre & 8 & 27 \\
Alguna vez & 19 & 63 \\
Rara vez & 1 & 3 \\
\hline Total & 30 & 100 \\
\hline
\end{tabular}

Tabla 3. Estrés Académico en estudiantes de Enfermería del VII semestre $(n=30)$.

\begin{tabular}{lcc}
\hline Características del Estrés & $\mathbf{X}$ & $\pm \mathbf{D E}$ \\
\hline Intensidad estrés académico & 81,30 & $\pm 3,10$ \\
Entorno estresor & 25,73 & $\pm 3,17$ \\
Reacciones físicas & 15,73 & $\pm 2,93$ \\
Reacciones psicológicas & 12,80 & $\pm 1,65$ \\
Reacciones comportamentales & 10,16 & $\pm 1,29$ \\
Afrontamiento del estrés & 18,93 & $\pm 2,26$ \\
\hline
\end{tabular}


Tabla 4. IE y EA de estudiantes de Enfermería de VII semestre (n=30).

\section{Estrés académico}

\section{Dimensión}

Rara vez Algunas Veces Casi siempre Siempre $\mathbf{P}^{*}$

\begin{tabular}{|c|c|c|c|c|c|c|c|c|c|c|}
\hline & & $\mathbf{N}^{\circ}$ & $\%$ & $\mathbf{N}^{\circ}$ & $\%$ & $\mathbf{N}^{\circ}$ & $\%$ & $\mathbf{N}^{\circ}$ & $\%$ & \\
\hline \multirow{8}{*}{$\begin{array}{l}\text { Inteligencia } \\
\text { Interpersonal }\end{array}$} & Marcd. Bajo & 1 & 50 & 0 & 0 & 1 & 50 & 0 & 0 & \multirow{8}{*}{$>0,05$} \\
\hline & Muy Bajo & 0 & 0 & 2 & 67 & 1 & 33 & 0 & 0 & \\
\hline & Bajo & 0 & 0 & 3 & 50 & 2 & 33 & 1 & 17 & \\
\hline & Promedio & 0 & 0 & 10 & 72 & 3 & 21 & 1 & 7 & \\
\hline & Alto & 0 & 0 & 3 & 75 & 1 & 25 & 0 & 0 & \\
\hline & Muy Alto & 0 & 0 & 1 & 100 & 0 & 0 & 0 & 0 & \\
\hline & Marcd. Alto & 0 & 0 & 0 & 0 & 0 & 0 & 0 & 0 & \\
\hline & TOTAL & 1 & & 19 & & 8 & & 2 & & \\
\hline \multirow{8}{*}{$\begin{array}{l}\text { Inteligencia } \\
\text { Intrapersonal }\end{array}$} & Marcd. Bajo & 0 & 0 & 8 & 67 & 3 & 25 & 1 & 8 & \multirow{8}{*}{$>0,05$} \\
\hline & Muy Bajo & 0 & 0 & 8 & 61 & 4 & 32 & 1 & 7 & \\
\hline & Bajo & 0 & 0 & 2 & 67 & 1 & 33 & 0 & 0 & \\
\hline & Promedio & 1 & 50 & 1 & 50 & 0 & 0 & 0 & 0 & \\
\hline & Alto & 0 & 0 & 0 & 0 & 0 & 0 & 0 & 0 & \\
\hline & Muy Alto & 0 & 0 & 0 & 0 & 0 & 0 & 0 & 0 & \\
\hline & Marcd. Alto & 0 & 0 & 0 & 0 & 0 & 0 & 0 & 0 & \\
\hline & TOTAL & 1 & & 19 & & 8 & & 2 & & \\
\hline \multirow{8}{*}{ Adaptabilidad } & Marcd. Bajo & 0 & 0 & 2 & 67 & 1 & 33 & 0 & 0 & \multirow{8}{*}{$>0,05$} \\
\hline & Muy Bajo & 0 & 0 & 6 & 55 & 3 & 27 & 2 & 18 & \\
\hline & Bajo & 0 & 0 & 7 & 70 & 3 & 30 & 0 & 0 & \\
\hline & Promedio & 1 & 20 & 3 & 60 & 1 & 20 & 0 & 0 & \\
\hline & Alto & 0 & 0 & 1 & 100 & 0 & 0 & 0 & 0 & \\
\hline & Muy Alto & 0 & 0 & 0 & 0 & 0 & 0 & 0 & 0 & \\
\hline & Marcd. Alto & 0 & 0 & 0 & 0 & 0 & 0 & 0 & 0 & \\
\hline & TOTAL & 1 & & 19 & & 8 & & 2 & & \\
\hline \multirow{8}{*}{ Manejo de estrés } & Marcd. Bajo & 0 & 0 & 0 & 0 & 0 & 0 & 0 & 0 & \multirow{8}{*}{$>0,05$} \\
\hline & Muy Bajo & 0 & 0 & 3 & 75 & 1 & 25 & 0 & 0 & \\
\hline & Bajo & 1 & 7 & 8 & 57 & 4 & 29 & 1 & 7 & \\
\hline & Promedio & 0 & 0 & 5 & 56 & 3 & 33 & 1 & 11 & \\
\hline & Alto & 0 & 0 & 3 & 100 & 0 & 0 & 0 & 0 & \\
\hline & Muy alto & 0 & 0 & 0 & 0 & 0 & 0 & 0 & 0 & \\
\hline & Marcd. alto & 0 & 0 & 0 & 0 & 0 & 0 & 0 & 0 & \\
\hline & TOTAL & 1 & & 19 & & 8 & & 2 & & \\
\hline \multirow{8}{*}{ Animo en General } & Marcd. Bajo & 0 & 0 & 3 & 50 & 2 & 33 & 1 & 17 & \multirow{8}{*}{$>0,05$} \\
\hline & Muy Bajo & 0 & 0 & 3 & 60 & 1 & 20 & 1 & 20 & \\
\hline & Bajo & 1 & 14 & 4 & 57 & 2 & 29 & 0 & 0 & \\
\hline & Promedio & 0 & 0 & 8 & 73 & 3 & 27 & 0 & 0 & \\
\hline & Alto & 0 & 0 & 1 & 100 & 0 & 0 & 0 & 0 & \\
\hline & Muy Alto & 0 & 0 & 0 & 0 & 0 & 0 & 0 & 0 & \\
\hline & Marcd. Alto & 0 & 0 & 0 & 0 & 0 & 0 & 0 & 0 & \\
\hline & TOTAL & 1 & & 19 & & 8 & & 2 & & \\
\hline
\end{tabular}

*Valor de p mediante prueba $\mathrm{chi}^{2}$. 
El que los estudiantes del estudio poseen una escasa IE en las dimensiones Inteligencia intrapersonal y Adaptabilidad, con puntajes muy bajos y que las dimensiones Inteligencia interpersonal y Estado de ánimo sean las más desarrolladas (Tabla 1), coinciden con Páez y Castaño ${ }^{(18)}$, quienes encontraron una deficiencia en la IE, representada, también, por las mismas dimensiones. Al respecto, es necesario mencionar que los bajos niveles de Adaptabilidad pueden indicar débiles motivaciones hacia el proceso enseñanza-aprendizaje y una actitud menos comprometida con la formación profesional. Por esto, es indispensable que el sistema educativo junto con estar dirigido a los aspectos cognoscitivos y técnicos, también esté orientado al desarrollo de otras habilidades, como la IE, siendo esta una dimensión elemental para una educación integral en el estudiante universitario. A pesar de las coincidencias anteriores, los resultados del actual estudio no coinciden con Vera, Espinoza, Piña y Segura ${ }^{(19)}$ o con Lavado ${ }^{(20)}$, pues los primeros encontraron que la mayoría de los estudiantes se encuentran en un rango de bueno-adecuado y el segundo encontró un nivel promedio de IE. Tales diferencias son fundamentales al considerar lo expresado por Goleman y Boyatzis ${ }^{(21)}$, quienes afirman que existen habilidades más importantes que la inteligencia académica a la hora de alcanzar un mayor bienestar laboral, personal, académico y social.

El proceso de formación profesional es una experiencia de aprendizaje que suele estar acompañada de estrés y requiere del estudiante un esfuerzo de adaptación. Lo anterior conlleva a cambios de estilo de vida y nuevos retos que implican preparación de trabajos académicos, interacción con docentes y compañeros de clases, trabajo en equipo y, primordialmente, administrar su tiempo.

La alta intensidad de EA en los estudiantes (puntaje promedio $=81,3$ ), coincide con Castillo, Barrios y Alvis ${ }^{(22)}$, quienes afirman que los estudiantes universitarios de la Carrera de Enfermería atraviesan por episodios de estrés ocasionado por tener que asumir ciertos roles académicos, que implica sobrecarga de actividades por las exigencias de las características propias de los programas del área salud, entre estos los de Enfermería. Estos serían muy demandantes en términos de tiempo, dedicación y recursos para el desarrollo de las diferentes actividades, situaciones todas que favorecerían el que los estudiantes universitarios de ciencias de la salud alcancen altos niveles de EA. Otros estudios que han detectado niveles de EA ya sea moderado ${ }^{(23)}$, elevado ${ }^{(24)} \mathrm{o}$ incluso niveles heterogéneos de estrés ${ }^{(22)}$ señalan que este estrés trae importantes consecuencias que pueden repercutir de forma negativa en la salud física y mental en los estudiantes del área de la salud $^{(25)}$. Belkis, Calcines y Monteagudo ${ }^{(26)}$ señalan que el EA es aquel generado por las demandas que impone el ámbito educativo y lo padecen los alumnos fundamentalmente de educación media y superior, cuya fuente exclusiva son los estresores relacionados con las actividades a desarrollar en el ámbito formativo, que impactan en su desempeño.

Por otra parte, Toribio y Franco ${ }^{(27)}$ definen EA como la situación que afecta el aprendizaje y el bienestar de los estudiantes, generada por las demandas altamente estresantes que impone la adaptación a un nuevo ambiente, las aulas universitarias. Este estrés de adaptación es el que caracteriza al EA y seguir una carrera universitaria significa tener propensión a desarrollar algún nivel de estrés que puede desencadenar problemas emocionales, cognitivos y fisiológicos en los que tanto estudiantes como docentes pueden verse afectados durante el desarrollo del proceso enseñanza-aprendizaje.

Todas estas afirmaciones confirman que el ámbito educativo y la complejidad de sus procesos son las principales fuentes de EA, fenómeno a partir del cual coexisten múltiples formas de conceptualizarlo, desde perspectivas educativas o psicológicas, sin obviar la imprescindible consideración biopsicosocial, inherente a lo que rodea al ser humano. Es un proceso de cambio, inmerso en el proceso enseñanza-aprendizaje que se expresa a través de un conjunto de mecanismos adaptativos, individuales e institucionales, producto de la demanda desbordada de las experiencias de los nuevos paradigmas educativos que se desarrollan en las instituciones de formación superior ${ }^{(28)}$.

Tanto la IE como el EA son temas relativamente nuevos en las ciencias de la salud y la información en la temática aún es limitada, por lo que, en el contexto de enfermería, se necesita entrenamiento para facilitar el desarrollo del bienestar personal entre los estudiantes de la carrera, con el propósito 
de disminuir la presencia de fuentes de estrés asociadas a su formación académica ${ }^{(29)}$. Para esto es fundamental abordar el desarrollo de la IE desde los primeros semestres de formación, con la finalidad de desarrollar destrezas emocionales y dotar al estudiante de recursos que permitan afrontar situaciones estresantes. Lo anterior mejora la calidad educativa y garantiza profesionales de enfermería preparados para afrontar nuevos retos.

Finalmente, es necesario indicar que la principal limitación del estudio es el tipo de diseño, pues su transversalidad exceptúa una evaluación de temporalidad y causalidad entre las variables evaluadas. Otro rasgo limitante puede atribuirse al uso de encuestas que contienen información referida por el participante, no siendo factible verificar la veracidad de la información brindada por los estudiantes.

\section{REFERENCIAS}

1. Hasegawa Y, Ninomiya K, Fujii K, Sekimoto T. Emotional intelligence score and performance of dental undergraduates. Odontology. 2016; 104 (3): 397-401.

2. Goleman D. Inteligencia emocional [Internet]. EEUU: Kairos; 1996 [citado 22 feb 2010]. 514 p.

Disponible en: https://books.google.com. pe/books/about/Inteligencia_emocional. html ? id =x 8 cTlu $1 \mathrm{rmA} 4 \mathrm{C} \& \mathrm{printsec}=$ frontcover\&source=kp_read_button\&redir_ esc $=\mathrm{y} \# \mathrm{v}=$ onepage $\& \mathrm{q} \& \mathrm{f}=$ false

3. Del Rosal SI, Dávila AM, Sánchez HS, Bermejo GM. La inteligencia emocional en estudiantes universitarios: diferencias entre el grado de maestro en educación primaria y los grados en ciencias. Int J Dev Educ Psychol [Internet]. 2016 [citado 22 nov 2017]; 2(1): 51-61. Disponible en: http:// dx.doi.org/10.17060/ijodaep.2016.n1.v2.176

4. Aparicio L, Escolano E, Rodríguez A, Soler J, Díaz O. Inteligencia Emocional y Bienestar II [Internet]. España: Universidad de San Jorge; 2016 [citado 5 dic 2017]. 860 p. Disponible en: https:// dialnet.unirioja.es/servlet/libro?codigo $=655308$

5. Márquez-Cervantes MC, Gaeta-González ML. Desarrollo de competencias emocionales en preadolescentes: el papel de padres y docentes. Rev Electrónica Interuniv Form del Prof [Internet]. 2017 [citado 10 dic 2017]; 20(2):221-35. Disponible

\section{CONCLUSIONES}

En esta investigación se concluye que no existe relación significativa entre Inteligencia emocional y frecuencia de Estrés académico en estudiantes del VII semestre de la Facultad de Enfermería de una universidad peruana. A pesar de ello, por la escasa Inteligencia emocional encontrada en relación con las dimensiones Intrapersonal y Adaptabilidad y el que las cinco dimensiones de la Inteligencia emocional están relacionadas con episodios de estrés en estudiantes que alguna vez presentaron esta situación, las investigadoras consideran que es necesario trabajar por mejorar la calidad educativa durante el proceso de formación profesional, enfocando las actividades educativas, no solo en la dimensión intelectual, también en el aspecto emocional de los estudiantes y desarrollar, desde los primeros años de la carrera, un proceso de enseñanza-aprendizaje de manera integral.

en: http://dx.doi.org/10.6018/reifop.20.1.232941

6. Borrachero A, Dávila MA, Costillo E, Mellado V. Las emociones del futuro profesorado de secundaria de ciencias y matemáticas, tras un programa de intervención. Ápice Rev Educ Científica [Internet]. 18 Jul 2017 [citado $22 \mathrm{feb}$ 2019]; 1(1): 17. Disponible en: http://revistas.udc. es/index.php/apice/article/view/arec.2017.1.1.2008

7. Farquhar JM, Kamei RK, Vidyarthi AR. Strategies for enhancing medical student resilience: student and faculty member perspectives. Int J Med Educ [Internet]. 2018 [citado 15 ene 2018]; 9(1): 1-6. Disponible en: http://creativecommons.org/ licenses/by/3.0

8. Domínguez EG. Inteligencia emocional e intensidad emocional en el juego motor [Internet]. España: Consejería de Educación; 2017 [citado 20 ene 2018]. 67 p. Disponible en: www.educarm.es/ publicaciones

9. Barraza J, Muñoz N, Behrens C. Relación entre inteligencia emocional y depresión-ansiedad y estrés en estudiantes de medicina de primer ańo. Rev Chil Neuro-Psiquiat. 2017; 55 (1): 18-25.

10. Garg R, Levin E, Tremblay L. Emotional intelligence: impact on post-secondary academic achievement. Soc Psychol Educ. 2016; 19 (3): 627-42.

11. Ramos E, Rodríguez A, Axpe I, Ferrara M. Perceived Emotional Intelligence and Life Satisfaction Among Adolescent Students: The 
Mediating Role of Resilience. J Happiness Stud [Internet]. 2018 [citado 22 ene 2018]; (0123456789): 1-18. Disponible en: http://link. springer.com/10.1007/s10902-018-0058-0

12. Birks Y, McKendree J, Watt I. Emotional intelligence and perceived stress in healthcare students: A multi-institutional, multi-professional survey. BMC Med Educ. 2009; 9(1): 1-8.

13. Cabanach RG, Souto-Gestal A, Fernández CR. Perfiles de regulación emocional y estrés académico en estudiantes de fisioterapia. Eur J Educ Psychol [Internet]. 1 dic 2017 [citado 2 feb 2019]; 10(2): 57-67. Disponible en: https://www.sciencedirect. com/science/article/pii/S1888899217300119

14. Chávez NU, Águila LP. La evaluación de la inteligencia emocional a través del inventario de Bar-On ICE: NA, en una muestra de nińos y adolescentes. Persona [Internet]. 2005 [citado 2 feb 2018]; 8: 11-58. Disponible en: https://dialnet. unirioja.es/servlet/articulo?codigo $=2872458$

15. Barraza MA. El Inventario SISCO del Estrés Académico. Investigación Educativa [Internet]. 2007 [citado 5 feb 2018]. Disponible en: https:// www.researchgate.net/publication/28175062_El_ Inventario_SISCO_del_Estres_Academico

16. Bryant SE, Malone TI. An Empirical Study of Emotional. Bus Educ Accreditation. 2015; 7(1): $1-11$.

17. Picasso Á, Lizano C, Anduaga S. Estrés académico e inteligencia emocional en estudiantes de odontología de una universidad peruana. Kiru [Internet]. 2016 [citado 22 feb 2019]. Disponible en: http://www.usmp.edu.pe/odonto/ servicio/2016/02/1020-3490-1-PB.pdf

18. Páez CM, Castaño CJ. Inteligencia emocional y rendimiento académico en estudiantes universitarios. Psicol desde el Caribe [Internet]. 2015 [citado 6 feb 2018]; 32(2): 268-85. Disponible en: http://www.redalyc.org/articulo. oa?id=21341030006

19. Vera A, Espinoza A, Piña L, Segura A. La inteligencia emocional en jóvenes universitarios: un estudio descriptivo. Psicol e Educ Present y Futur [Internet]. 2016 [citado 12 mar 2018]; (C33.5): 1288-1291. Disponible en: https:// rua.ua.es/dspace/bitstream/10045/64530/1/ Psicologia-y-educacion_151.pdf

20. Lavado RA. Inteligencia Emocional en Estudiantes de Enfermería de un Instituto Superior Tecnológico en Puente Piedra [Tesis de maestría]. Perú: Universidad Ricardo Palma; 2018. 74 p. Disponible en: http://repositorio.urp.
edu.pe/bitstream/handle/URP/1429/Tesis.RALP. pdf? sequence $=1 \&$ is Allowed $=y$

21. Goleman D, Boyatzis RE. Emotional Intelligence Has 12 Elements. Which Do You Need to Work On? Harvard Business Review [Internet]. 2017 [citado 13 mar 2018]; 1-5. Disponible en: https:// hbr.org/2017/02/emotional-intelligence-has-12elements-which-do-you-need-to-work-on

22. Castillo Y, Barrios A, Alvis L. Estrés académico en estudiantes de enfermería de Cartagena, Colombia [Internet]; 2018 [citado 8 mar 2018]; 20(2). Disponible en: https://revistas.javeriana. edu.co/files-articulos/IE/20-2\%20(2018II)/145256681002/145256681002_visor_jats.pdf

23. De Dios DJM, Varela MI, Braschi DL, Sánchez ME. Estrés en estudiantes de enfermería. Educ Médica Super [Internet]. 2017 sep [citado 8 mar 2018]; 31(3): 110-23. Disponible en: http://scielo. sld.cu/scielo.php?script=sci_arttext\&pid=S086421412017000300013\&lng=es.

24. Pimienta CC, Chacón T, Cruz D. Ansiedad y fuentes de estrés académico en estudiantes de carreras de la salud. Investig en Educ médica. 2016; 5(20): 230-7.

25. Jerez-Mendoza M, Oyarzo-Barría C. Estrés académico en estudiantes del Departamento de Salud de la Universidad de Los Lagos Osorno. Rev chil neuro-psiquiatr [Internet]. 2015 Sep [citado 31 oct 2018]; 53(3): 149-157. Disponible en: https:// scielo.conicyt.cl/pdf/rchnp/v53n3/art02.pdf

26. Belkis AÁ, Calcines CM, Monteagudo DLGR. Estrés académico. Edumecentro [Internet]. 2015 [citado 20 dic 2018]; 7(2): 163-78. Disponible en: http://www.revedumecentro.sld.cu/index.php/ edumc/article/view/530/pdf_77

27. Toribio-Ferrer C, Franco-Bárcenas S. Estrés Académico: El Enemigo Silencioso del Estudiante. Salud y Adm. 2016; 3(200): 11-8.

28. Román CC, Hernández RY. El estrés académico: una revisión crítica del concepto desde las ciencias de la educación. Rev Electrónica Psicol Iztacala [Internet]. 2011 [citado 20 nov 2018]; 14(2): 1-14. Disponible en: http://www.revistas.unam.mx/ index.php/repi/article/view/26023/24499

29. Pulido-Martos M, Augusto-Landa JM, LópezZafra, E. Estudiantes de Enfermería en prácticas clínicas: El rol de la inteligencia emocional en los estresores ocupacionales y bienestar psicológico. Index Enferm [Internet]. 2016 [citado 12 dic 2018]; 25(3): 215-9. Disponible en: http://scielo. isciii.es/pdf/index/v25n3/academia.pdf 